\title{
İçortam Görünür Işık Haberleşme Kanallarında Güç Analizi
}

\author{
Asuman Savaşcıhabeş* \\ * Nuh Naci Yazgan Üniversitesi, Mühendislik Fakültesi, Elektrik-Elektronik Mühendisliği Bölümü, Kayseri, Türkiye, (ORCID: 0000-0002-7261-1906), \\ ahabes@nny.edu.tr \\ (International Conference on Design, Research and Development (RDCONF) 2021 - 15-18 December 2021)
}

(DOI: 10.31590/ejosat.1040234)

ATIF/REFERENCE: Savaşcıhabeş, A. (2021). İçortam Görünür Işık Haberleşme Kanallarında Güç Analizi. Avrupa Bilim ve Teknoloji Dergisi, (32), 536-541.

$\ddot{O} \mathbf{z}$

Optik kablosuz haberleşme teknolojisi, radyo frekansı iletişiminin önemli bir alternatifi olarak ortaya çıkmıştır. RF iletişimdeki bandgenişliğinin sınırlı olması nedeniyle, kullanıcıların yüksek hızlı kablosuz iletişim sistemlerine olan ihtiyacı artmaktadır. RF sisteminin kontrolsüz radyasyon, sınırlı bandgenişliği, düzenlenmiş frekans ve mobil kullanıcı sayısının artması gibi birtakım kısıtlamaları nedeniyle, RF tabanlı iletişim son yıllarda spektrum darlı̆̆ sorunuyla karşı karşıya kalmıştır. RF’ten farklı olarak görünür ışık haberleşmesi (VLC), iletim kanalları için görünür ışık spektrum tayfında daha yüksek frekans bantları tercih edilir. Band genişliği avantajı nedeniyle VLC için görünür ışık frekans bandı kullanılmakta, bu sayede yüksek aktarım hızı ve elektromanyetik girişime dayanıklı olduğu için büyük avantaj sağlanmaktadır. Bu açıdan optik kablosuz iletişim, yeni nesil kablosuz iletişim sistemleri için umut verici bir çözüm olarak değerlendirilmektedir. Bu çalışmada VCL sistemlerinin tek dallı doğrudan görüş (Line-of-sight, LOS) düz sönümlü kanallarda güç analizi incelenmiş, kanal darbe cevabı ve optik güç dağılımları analiz edilmiş̧tir.

Anahtar Kelimeler: Görünür Işık Haberleşmesi, Optik Kablosuz İletişim, Güç Analizi.

\section{Power Analysis for Indoor Visible Light Communication Channels}

\begin{abstract}
One of the most important alternative techniques for radio frequency communications is optical wireless communication technology. Due to the RF bandwidth limitation, the need for high speed wireless communication systems has been increasing in recent years. Due to the limitations of the RF system such as uncontrolled radiation, limited bandwidth, regulated frequency, and the increase in the number of mobile users, RF-based communication has faced a serious spectrum problem in recent years. Visible light communication (VLC) uses higher frequency bands in the visible light spectrum for the transmission medium. Considering the visible light spectrum due to its bandwidth advantage, VLC is advantageous as it offers high transmission speed and strong resistance to electromagnetic interference. In this respect, optical wireless communication is considered as a promising solution for new generation wireless communication systems. In this paper we investigate channel impulse response and power analysis of VLC systems over single-tap Line-of-sight (LOS) flatfading channels.
\end{abstract}

Keywords: Visible Light Communications, Optical Wireless Communication, Power Analysis.

\footnotetext{
*Sorumlu Yazar: ahabes@nny.edu.tr
} 


\section{Giriş}

Görünür 1şıkla haberleşme (VLC) son y1llarda radyo iletişimine ve kızılötesi haberleşmeye alternatif olarak popüler hale geldi. Görünür 1şı̆̆ın elektromanyetik dalgalardan etkilenmeyen band genişliğine sahip olması bu alternatifi radyo frekansından ayıran bir özellik olarak karşımıza çıkmaktadır (Fiorarli, 2014).

Son yıllarda akıllı cihazların yaygınlaşması ve yeni nesil Nesnelerin İnterneti (IoT) uygulamalarının hızla büyümesiyle birlikte yeterli bant genişliği arayışı hızla artmaktadır. Kablosuz kanallardaki veri ve ses transferinin $\% 70$ oranında kapalı ortamda gerçekleştirği bilinmektedir. Sorunsuz bir iç mekan kablosuz deneyimi sağlamak için özellikle düşük maliyetli ve son derece güvenilir çözümler gereklidir. Görünür 1ş1k iletişimi (VLC) ile bu amaca yönelik çözümler gerçekleştirilebilir ve kablosuz iletişim amaçları için mevcut aydınlatma altyapısından (LED'ler kullanılarak) faydalanılmaktadır (Xu, 2015). VLC ile ilgili literatürün yanı sıra artan akademik ve endüstriyel ilgiye rağmen, uygun kanal modellerinin geliştirilmesine ihtiyaç duyulduğu görülmektedir. Mevcut literatürde, daha önceleri kızılötesi (IR) frekansları için geliştirilmiş kanal modelleri (Song, Pincemin, Vgenopoulou, Roudas, Amhoud, \& Jaouen, 2015), net bir gerekçe olmaksızın VLC sistemlerinin performans değerlendirmesi için de kullanılmaktadır (Zhang, 2017). Ancak görünür 1 şık ve IR bantlarının farklı özellikler gösterdiği bilinmektedir ve bu durum VLC kanal modellerinin geliştirilmesini zorunlu kılmaktadır (Marcus \& Pattan, 2005).

Literatürde görünür 1şıkla haberleşme kanal modelleri üzerine birtakım çalışmalar yapılmakta ve farklı senaryolar için kanal dürtü cevapları bulunmaktaktadır (Kahn, Krause, \& Carruthers, 1995). Bu çalışmalarda bir çeşit ticari olarak dağıtılıan optik ve aydınlatma sistemi tasarımı yazılımları kullanılarak kanal dürtü yanıtları sunulmuştur. Ardışıl olarak ve ardışıl olmadan ışın izleme yapabilen optik ve aydınlatma tasarımı kullanılan bu çalışmalarda kullanıcının tsasrlamışı olduğu bir kapalı alandaki LED tarafından yayılan ışınların etkileşimini hassas biçimde tanımlamak mümkün olmaktadır (He, Cao, \& Zou, 2011). Ardış1l olmayan durumda ise 1şınlar bir nesneye ulaşıncya kadar fiziksel olarak tanımlanan bir hat üzerinde ilerlemektedir. İletim ortamında görüş hattı (LOS) ve ortamdaki zemin, duvarlar ve tavan dan yansımalar ile alıcıya ışınlar ulaşmaktadır. Burada kapalı ortamın boyutları önem kazanmakta olup alıcı ile göndericilerin ortamdaki konumları ve malzemelerin türü önem kazanmaktadır (Ramaswami, 2002). Malzemeler arasında kımızı tuğla, çam kerestesi, siyah parlak boya, pencere camı ve duvar sıvası gibi materyallerin yansıma değerleri NASA web sistesinde yer almaktadır (Aster Spectral Library-Version 2.0, 2021). VLC'nin avantajları arasında daha fazla spektrum aralığı kullanabilme, RF ile karşılaştırıldığında 100 kat fazla band genişliği, yüksek enerji verimliliği, yüksek veri güvenliği, RF'nın bulunmadığı alanlarda erişilebilirlik avantajları yer almaktadır (Lee, Park, \& Barry, 2011).

$\mathrm{Bu}$ çalışmada, kablosuz iletişimde OWC sistemleri incelenmiş. Fiziksel katman algoritmaları kullanılarak yüksek hızda data iletimine izin veren, dış etkenler nedeniyle fazla bozunuma uğramayan ve görünür 1şıkla iletişim yapan iletişim sistemlerinin temel yapıları üzerine araştırmalar sunulmaktadır. Görünür 1ş1k haberleşmesinde önemli bir konu olan güç analizi incelenmiştir. Optik kablosuz haberleşmenin temel prensipleri ile optik 1şık kaynakları ve dedektörler ikinci bölümde sunulmuştur. Üçüncü bölümde bilgisayar benzetim çalışmaları yer almakta olup, dördüncü bölümde sonuçlar ve değerlendirme verilmiştir.

\section{Kanal Modelinin Karakterize Edilmesi}

Tam çift yönlü mod OWC sistemleri için, kanalları elde etmek amacıyla çok-yollu girişim kanalları, çok-kullanıcılı erişim ve ağ şemaları kullanımında en etkin kanal modelinin seçilmesi önem taşımaktadır (Elgala, Mesleh, \& Haas, 2010). Etkin optik sistem iletişimini sağlamak, kanal özelliklerinin iyi anlaşılması için uygulamak ve işletmek. İç mekan optik kablosuz iletişiminde geniş kullanım alanı bulunan birçok topoloji mevcuttur. Bu kapsamda, iç mekan konfigürasyonlarında dikkate alınacak bir dizi parametre dikkatlice seçilmelidir. İletim ortamında bulunan vericilerin yönlülüğü, gönderici ve alıcılar arasında doğrudan bir görüş hattının (LOS, line of sight) bulunmasına bağlı olarak şekillenebilmektedir (Ghassemlooy, Popoola, \& Rajbhandari, 2019).

Tablo 1. Kablosuz İletişim Teknolojileri (Ghassemlooy, Popoola, \& Rajbhandari, 2019)

\begin{tabular}{l|l}
\hline Teknoloji & Kablosuz Veri Hızı (bps) \\
\hline Wi-Fi (IEEE 802.11n) & $150 \mathrm{Mbps}$ \\
\hline Bluetooth & $3 \mathrm{Mbps}$ \\
\hline IrDa & $4 \mathrm{Mbps}$ \\
\hline & Future \\
\hline Wi-Gig(IEE 802.ad) & $2 \mathrm{Gbps} @ 60 \mathrm{GHz}$ \\
\hline White Wi-Fi & $24 \mathrm{Mbps} @ 54 \mathrm{ve}$ \\
(IEEE 802.11af \& IEEE & $790,900 \mathrm{MHz}$ \\
802.11ah) & \\
\hline Giga-IR & $1 \mathrm{Gbps}$ \\
\hline LiFi & $>10 \mathrm{Gbps}$ (in a room) \\
\hline
\end{tabular}

Optik kablosuz iletişim (Optik Kablosuz İletişim, OWC), son otuz yıldır piyasada bulunan RF kablosuz sistemlerini tamamlayıcı yenilikçi bir teknoloji, sabit iç ve dış mekan analizlerinde düşük gecikme zamanı ve düşük maliyetle yüksek kapasite geliştirmek üzere uyarlanabilmektedir. IoT, büyük veri ve bulut kullanımıyla üretilen veri miktarı, mevcut haberleşme kalitesini artırmaya yönelik değişim gösterecektir. Böylece, tüm kanallar sinyal spektrumu tarafindan tamamen işgal edilirse, telekomünikasyon altyapısının hıza ayak uydurup uyduramayacağı ve servis sağlayıcıların iletim kapasitelerini artırıp artıramayacağıdır (Ricklin, Hammel, Eaton, \& Lachinova, 2006).

\subsection{Optik Kaynaklar ve Detektörler}

$5 \mathrm{G}$ ve ötessi haberleşme için popüler olan görünür 1 ş1k iletişiminin temeli, iletişim için radyo dalgaları bandını kullanmak yerine görürnür 1ş1k spektrumunu kullanmaktır. VLC sistem tasarımında verici olarak LED 1şıkları kullanılmakta olup, optik kanal geleneksel RF kanal yapısından tamamen farklıdır. LED'ler ve LD kullanımı ile, elektromanyetik spektrumun görünür bölgesinden kızılötesi (IR) frekans spektrununa doğru çok çeşitli dalga boylarında veya renklerde yayınım sağlanır (Moreira, Valadas, \& Duarte, Optical interference produced by artificial light, 1997). Sistem yapısında alıcı olarak fotodiyot kullanılmaktadır (Street, Stavrinou, Obrien, \& Edwards, 1997). Tablo 2 görünür 1şıkla haberleşmenin RF ve IR teknlolojileri ile karşılaştırmasını içermektedir. 
Tablo 2. Görünür Işsıla Haberleşmenin(VLC) RF ve IR teknolojileri ile karşıllaştırılması

\begin{tabular}{l|l|l|l}
\hline Nitelikler & VLC & IR & RF \\
\hline Elektromanyetik Girişimler & Hayır & Hayır & Evet \\
\hline LOS & Evet & Evet & Hayır \\
\hline Hizmet & Aydınlatma ve iletişim & İletişim & İletişim \\
\hline Kapsama & Dar & Dar & Geniş \\
\hline Hareketli Kullanım & Sinırlı & Sınırlı & İyi \\
\hline Güç Tüketimi & Az & Az & Orta \\
\hline
\end{tabular}

\subsubsection{Işıık Yayan Diyot (LED) Kullanımı}

LED, elektronik stimülasyona uğradığında kendiliğinden emisyon yoluyla tutarsız bir ışık yayar. Elektronik uyarım, pn bağlantısı boyunca hem ileri hem öngerilim uygulanarak elde edilir. Foton emisyon hızı ve dalga boyu şu şekilde hesaplanır:

$$
\begin{gathered}
I(E=h f) \infty \sqrt{E-E_{g}} \exp \left(-\frac{E}{k T}\right), E=E_{2}-E_{1}=h f=\frac{h c}{\lambda} \\
\lambda=\frac{h c}{E(e V)} \mu m
\end{gathered}
$$

Burada $E_{\mathrm{g}}$ yariiletken elemanın içi boş bant enerjisi ve $\mathrm{k}$ ise Boltzmann sabiti ve ayrıca T sıcaklığı ifade etmektedir[11].

Dalga boyu şu şekilde hesaplanır:

$$
\Delta \lambda=\frac{1.8 \mathrm{kT}}{\mathrm{hc}}
$$

Genel olarak, iç mekan OWC sistemi, kaynak ve geniş alanlı fotodetektörler olarak bir LED kullanır. Radyasyon yoğunluğu modelinin açısal dağılımı, aşağıda verilen dağılımla genelleştirilmiş bir Lambert radyan yoğunluğu kullanılarak modellenmiştir.

$$
R_{O}(\varnothing)=\left\{\begin{array}{cc}
\frac{\left(m_{l}+1\right)}{2 \pi} \cos ^{m_{l}}(\phi), & \phi \in[-\pi / 2, \pi / 2] \\
0, & \phi \geq \pi / 2
\end{array}\right.
$$

Burada, $m_{l}$, kaynak ışının yönlülüğünü ifade eden Lambert'in mod derecesi olmaktadır ve $\emptyset=0$ değeri ise yayılan gücün maksimum açısıdır.

$$
m_{l}=\frac{-\ln 2}{\ln \left(\cos \emptyset_{1 / 2}\right)}
$$

Lambertian emisyon $\mathrm{m}_{\mathrm{l}}$ derecesi, aşağıda verilen formülde ifade edildiği gibi yarı güçteteki yani $\emptyset_{1 / 2}$ 'deki LED yarım açısı ile bağlantılıdır.

\subsubsection{Kanal Modeli}

Tam çift yönlü mod optik kablosuz haberleşme sistemleri için, kanalları elde etmede çok-yollu girişim, çok-kullanıcılı erişim girişımi ve ağ yapıları tanımlanırken doğru bir kanal yapısı kullanılması gereklidir. OWC sisteminin giriş-çıkış parametreleri aşağıdaki gibi temsil edilir:

$$
y(t)=G_{o c} R x(t) \otimes h(t)+n(t)
$$

$$
y(t)=\int_{-\infty}^{\infty} \operatorname{Rhx}(\tau) h(t-\tau) d(\tau)+n(t)
$$

Burada $\otimes$ konvolüsyon operatörüdür, G_oc ise optik alıcının kazancıdır. R, fotodedektöre gelen 1şığı̄n spektral güç dağılımına bağlıdır ve fotodedektörün dalga boyuna göre değişim gösteren bir tepki fonksiyonudur.

Aynı zamanda R fotodetektör yanıtıdır, $h(t)$ temel bant kanal darbe yanıtıdır ve $n(t)$, çift yönlü güç spektral yoğunluğu ile Beyaz Gauss gürültüsü (AWGN) nün toplamıdır (Lomba, Valadas, \& Duarte, 2000).

$\mathrm{Bu}$ ifadelerdeki $\mathrm{h}(\mathrm{t})$, iç ortam optik kablosuz haberleşme kanallarında çok yollu dağıtımın etkilerini içeren optik kablosuz ortam kanal fonksiyonudur:

$$
h(t)=f(x)=\left\{\begin{array}{c}
\frac{2 t_{0}}{t^{3} \sin ^{2}(F O V)} t_{0} \leq t \leq \frac{t_{0}}{\cos (F O V)} \\
0, \text { diğer }
\end{array}\right.
$$

burada $\mathrm{h}(\mathrm{t})$ minimum kanal zaman gecikmesidir. Eşdeğer model, temel bant OWC kanalında yani optik iletişimde $\mathrm{x}(\mathrm{t})$ iletilen sinyal genliğinden çok sinyalin gücünü tanımlar ve verici tarafındaki sinyal gücü için $\mathrm{x}(\mathrm{t})$ pozitif değerli olmalıdır (Elgala, Mesleh, \& Haas, 2010).

İçortam OWC için bağlantı uzunluğu RF'e nispeten daha kısadır ve bu nedenle optik kablosuz sistemlerde zayıflama, emilim ve saçılma çok düşüktür (Hayasaka \& Ito, 2007).

Şekil 1.'de gösterilmiş olan bir Lambertian kaynağına sahip OWC bağlantısı ile iletim sisteminde, $\mathrm{T}_{\mathrm{s}}(\psi)$ optik band geçiş filtresine sahip ve kazancı $g_{s}(\psi)$ olan bir gönderici-alıcı senaryosu dikkate alındığında "d" mesafesinde ve farklı açılar için kanal parametresine ait alıcının kazancı aşağıdaki denklem ile hesaplanır:

$$
\begin{gathered}
H_{L O S}(0)= \\
\left\{\begin{array}{c}
\frac{\mathrm{A}_{r}\left(m_{l}+1\right)}{2 \pi \mathrm{d}^{3}} \cos ^{m_{l}}(\varnothing) T_{s}(\psi) g(\psi) \cos (\psi), 0 \leq \psi \leq \psi_{c} \\
0, \text { diğer }
\end{array}\right.
\end{gathered}
$$

Burada, alıcı gücü aşağıdaki denklem ile belirlenir:

$$
P_{r-L O S}=H_{L O S}(0) P_{t}
$$

LOS bağlantısındaki verici-alıcı konfigürasyonu için LOS sinyalin artışı ise aşağıdaki denklem ile hesaplanmaktadır:

$$
H_{L O S}\left(m_{l}\right)=\frac{\left(m_{l}+1\right)}{2} H_{L O S}
$$


Burada $\mathrm{H}_{\mathrm{LOS}}\left(\mathrm{m}_{\mathrm{l}}\right)$ ise $\mathrm{m}_{\mathrm{l}}=1$ değerine sahip Lambertian göndericiyi ifade etmektedir.

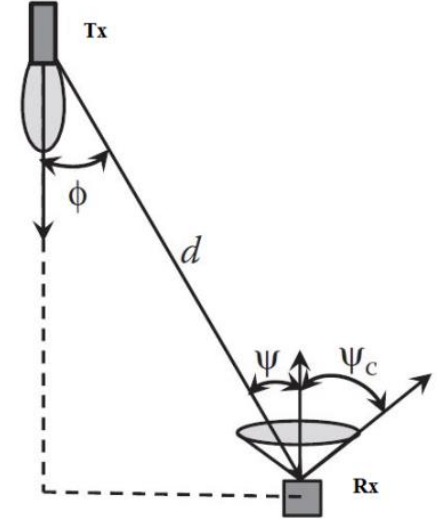

Şekil 1. LOS Propagasyon modeli (Tx:gönderic, Rx:alıcı)

\subsubsection{Güç Analizi}

Sistem performansına bakıldığında, iç mekan optik kablosuz kanallarda AWGN gürültüsüne ek olarak yapay $1 s ̧ 1 \mathrm{k}$ kaynaklarından ileri gelen periyodik ve deterministik gürültü formları da mevcuttur ve bu gürültü sinyalleri birleşerek bağlantı performansını büyük oranda düşürmektedir (Kahn \& Barry, 1997). İç ortam haberleşmesi çok-yollu semboller arası girişimden (ISI:inter-symbol interference) olumsuz etkilenmektedir (Moreira, Valadas, \& Duarte, Optical Interference produced by artificial light, 1997). Bu nedenle örneğin tipik bir iç ortam OWC sistemi için $260 \mathrm{Mbps}$ gibi erişilebilir maksimum veri hızını sınırlamaktadır (Gfeller \& Bapst, 1979).

Görünür 1 şık haberleşmesinde, bu sistemler için yaygın olarak kullanılan modülasyon tekniklerinden renk kaydırmalı anahtaralama modülasyonunun (CSK, color shift keying) sistem performansina olan etkisi bit-hata-oranı analizleri cinsinden (Tekin, Savaşcrhabeş, \& Ertuğ, 2021)' de gösterilmiştir. Işık kaynaklarının OWC sistem performansı üzerindeki etkileri incelendiğinde, özellikle kapalı ortamlarda kullanılan alıcı-verici yapılarının hem doğal hem de yapay kaynaklardan yayılan yoğun ortam 1şı̆̆ına maruz kalmaları nedeniyle ciddi performans düşüşüne neden olduğu görülmektedir (Narasimhan, Audeh, \& Kahn, 1996). Bu arka plan radyasyonunun ortalama gücü, beyaz Gauss ve alınan sinyalden bağımsız olarak doğru bir şekilde modellenen atış gürültüsü (shot noise) üretmektedir (Ghassemlooy, Hayes, \& Wilson, 2003).

$\mathrm{Bu}$ çalışmada aç-kapa anahtarlama (OOK:on-off keying), sayısal darbe modülasyonu (DPIM: digital pulse internal modulation) ile sembollerarası girişim varken hata olasılığ dahil edilerek normalize optik güç gereksinimine karşın veri hızının değişimi incelenmiştir. Gürültüyü gidermek için herhangi bir filtreleme veya diğer kompanzasyon teknikleri dahil edilmediği senaryo için normalize optik güç gereksinimi (NOGG) için veri hızı değişimi simülasyon analizleri ile ortaya konulmuştur. Burada farklı kanal şartları altında farklı modülasyon tekniklerini karşılaştırmak için aşağıda verilen temel performans göstergesi NOGG kullanılmıştır. Bir sistemin NOGG'si girişim yapan kanalda istenen bit/slot hata olasılığını

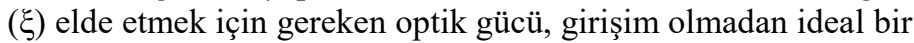
AWGN kanalda 1 Mbps'da OOK sistemininkiyle normalize ederek hesaplanır:
NOGG $=\frac{\xi \text { elde etmek için gereken optik güç }}{\text { ideal AWGN kanalda } \xi \text { elde etmek için gereken optik güç }}$

$\mathrm{Bu}$ çalışmada tüm simülasyonlarda, gerçek simüle edilmiş düşük ve yüksek frekanslı girişim istenen veri sinyaline eklenir. Bununla birlikte, simüle edilmiş sinyalin süresi, düşük frekanslı bileşenin bir tam döngüsünden daha kısa olabilir. Bu nedenle simülasyonlar düşük rekans döngüsü sırasında rastgele bir noktada başlamaktadır. Çalışmada $10^{-6}$ hata olasılığı standart olarak alınmış ve NOGG hesaplamasında kullanılmıştır.

\section{Bilgisayar Benzetim Sonuçları}

Çıkış sinyalinin frekans ve zaman domeninde normalizasyonu ise sirasıyla Şekil.2'de ve Şekil.3'te gösterilmiştir. LOS kanalda elde edilen performans yansımalı iletime göre daha iyi sonuç göstermiştir.

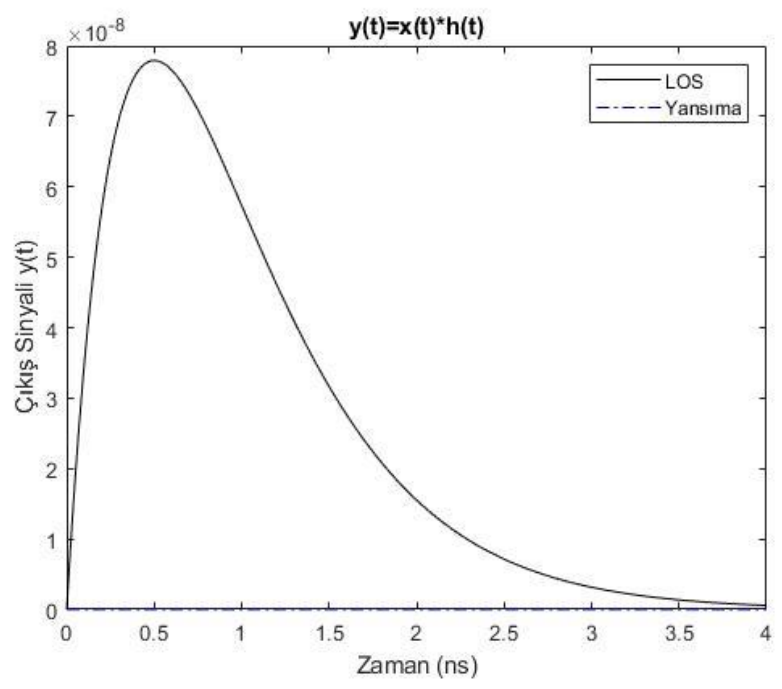

Şekil 2. Çıkış Sinyali (zaman domeni değişimi)

İçortam haberleşmesinde alıcıda nominal güç dağılımı ise Şekil.4'te verilmiştir. Burada optik alıcı kazancı normalize edilmiştir. LOS ve çok yollu iletimdeki yansıma etkisinde çıkış sinyalinin frekans artışına karşın değişimi gösterilmektedir.

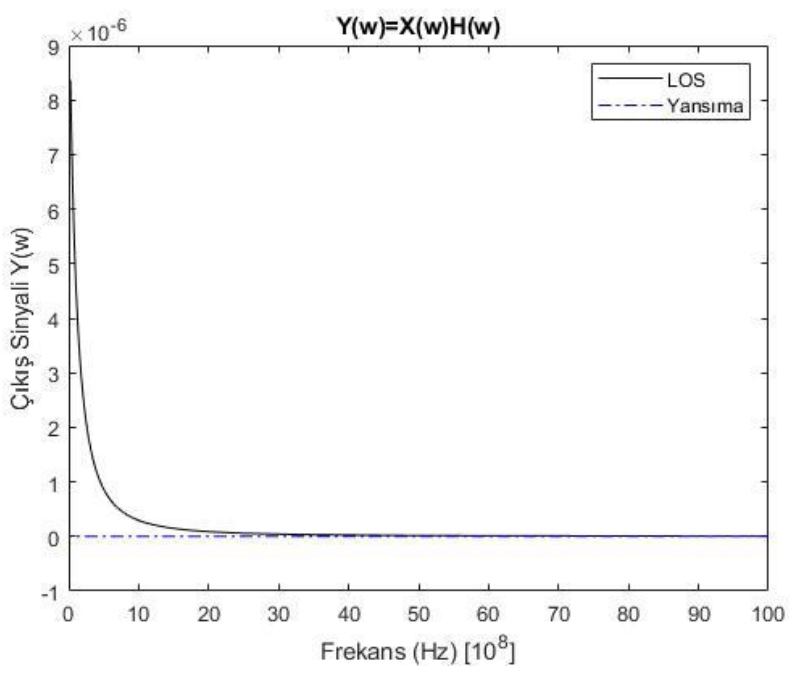

Şekil 3. Çıkış Sinyali (frekans domeni değişimi) 


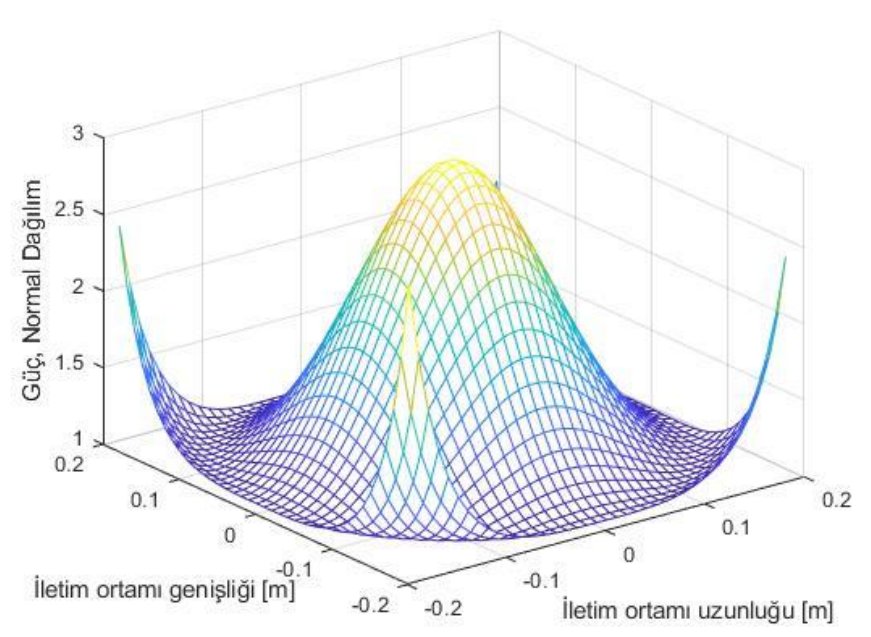

Şekil 4. Alıcı Nominal Güç Dăğllımı

İçortam haberleşmede iletim kanalının uygun senaryoda ortam uzunluğu ve ortam genişliğine göre güç değişim analizi Şekil.5'te verilmiştir. Buna göre, LOS yolunun modellendiği $(0,0)$ boyutları için iletim ortamında maksimum güç erişimi sağlandığı açıkça gösterilmektedir.

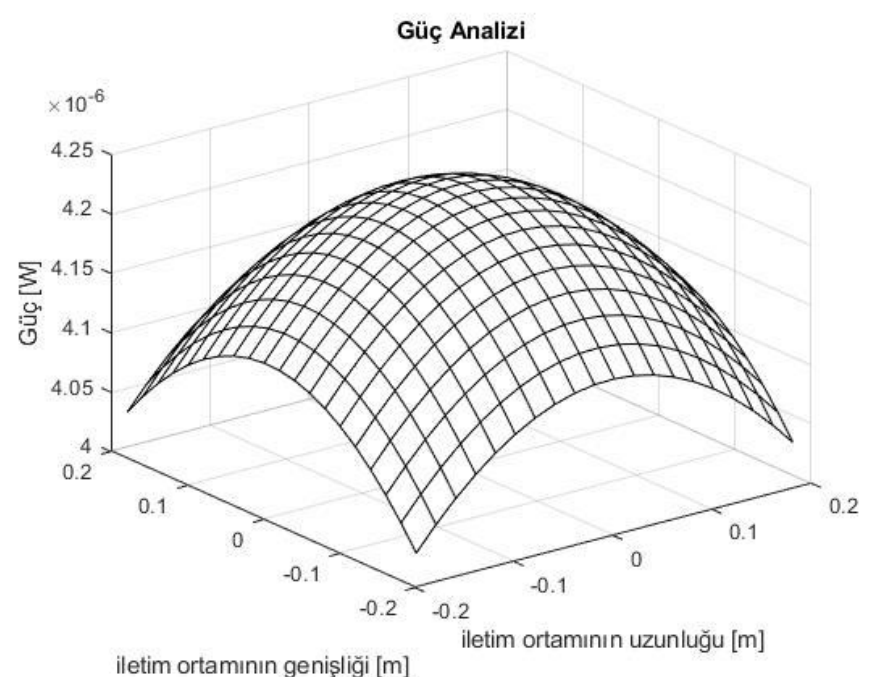

Şekil 5. Illetim ortamında VLC Sistemin Güç Analizi

Şekil 6.'da veri hızının değişimine karşın sayısal darbe girişim modülasyonunun (DPIM) farklı modülasyon derecelerine ait $(4,8,16)$ girişimli ve girişimsiz kanallarda normalize optik güç gereksinimlerine karşın veri hızı değişimleri incelenmiş ve kodlama yapılmayan düz sönümlü kanal için Matlab $(\underset{C}{ }$ programında elde edilen sonuçlar çizdirilmiştir. Burada baskın gürültü kaynağının floresan ışık girişimi olduğunu gösteren 1 ila 200 Mbps veri hızları için NOGG'de değişim oranı yüksek değildir. Ayrıca Şeklil 6'dan açıkça görüleceği üzere, maksimum veri hızı olan 200Mbps için 8-DPIM modülasyonunda erişilen NOGG değeri $14 \mathrm{~dB}$ olarak elde edilmiştir. Bu değer, 16-DPIM ölçümünün $2 \mathrm{~dB}$ üzerinde ve 4-DPIM değerinin ise $2 \mathrm{~dB}$ altındadır. Girişimsiz kanallarda ise aynı veri hızlarına daha düşük SNR seviyelerinde erişildiği görülmektedir. Şekil 6'da görüldüğü üzere $10 \mathrm{Mbps}$ veri hızına girişimsiz kanalda 4-DPIM modülasyonu altında $5 \mathrm{~dB}$ ile erişim sağlanırken, aynı veri hızı girişimli kanalda eşit modülasyon şartlarında $15 \mathrm{~dB}$ seviyesinde gözlenmektedir.

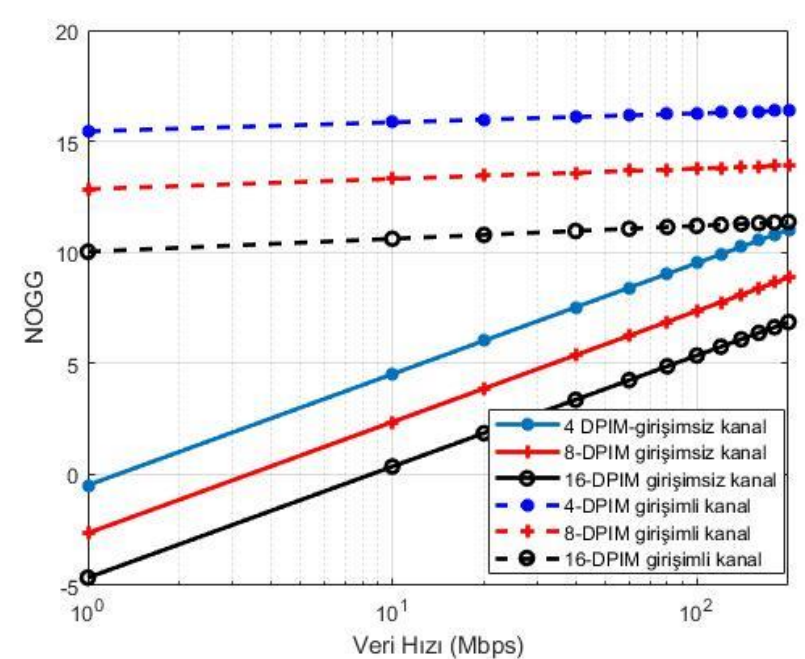

Şekil 6. 4-DPIM, 8-DPIM ve 16-DPIM Modülasyonlarında girişimli ve girişimsiz kanallar için Normalize Optik Güç Gereksinimine karşın veri hızının değişimi

\section{Sonuç}

VLC, radyo frekans dalgalarına alternatif olarak kullanılacak yeni bir görünür 1 şı altyapısına sahip olmakla birlikte, artan bant genişliği talebini karşılamak için yüksek iletim kapasitesine ulaşma ve sistemlerin yüksek frekans bant genişliğine ulaşma avantajları ile yeni nesil iletişim sistemlerinde gelecek vaat eden uygulamalardan biridir. Optik Kablosuz İletişim sistemleri ve dahilinde yer alan Görünür Işsk İletişimi (VLC), son yıllarda üzerinde çalışmaların yapıldı ̆̆ iletişim alanı araştırması olarak karşımıza çıkmaktadır. Bu çalışmada, optik kablosuz haberleşmenin temel prensipleri, optik ışık kaynakları ve dedektörler ile OWC kanal modeli üzerine yapilan incelemelerden elde edilen bilgiler sunulmuş, sistem çıkışının zaman ve frekans domeni değişimi ile alıcıda nominal güç değişimi analizleri yapılmıştır. Ayrıca DPIM modülasyonun kullanıldığı girişimli ve girişimsiz düz sönümlü kanallarda veri normalize güç gereksinimine karşın veri hızı artışı incelenmiş, $14 \mathrm{~dB}$ seviyelerinde yaklaşık $200 \mathrm{Mbps}$ veri hızlarına ulaşıldığ sonucuna ulaşılmış, bunun sonucunda ise kısıtlı spektrum dezavantajına sahip olan RF'e alternatif olarak geliştirilen OWC sistemlerinde VLC kullanımı ile girişimli ve girişimsiz kanallarda yüksek veri hızlarına erişilebileceği ortaya konulmuştur.

\section{Kaynakça}

Aster Spectral Library-Version 2.0. (2021). 10 6, 2021 tarihinde nasa.gov: http://speclib.jpl.nasa.gov adresinden alınd1.

Elgala, H., Mesleh, R., \& Haas, H. (2010). An LED Model For Intensity-Modulated Optical Communication Systems. IEEE Photonics Technology Letters, 22(11), 835-837.

Fiorarli, M. (2014). Challenges for 5G Transport Networks. IEEE 2014 International Conference on Advanced Networks and Telecommunications Systems. New Delhi.

Gfeller, F., \& Bapst, U. (1979). Wireless in-house data communication via diffuse infrared radiation. Proceedings of the IEEE, 67(11), 1474-1486. 
Ghassemlooy, Z., Hayes, A., \& Wilson, B. (2003). Reducing the effects of intersymbol interference in diffuse DPIM optical wireless communications. IEE Proceedings Optoelectronics, 445-452.

Ghassemlooy, Z., Popoola, W., \& Rajbhandari, S. (2019). Optical Wireless Communications. Florida, ABD: CRC Press.

Hayasaka, N., \& Ito, T. (2007). Channel modeling of nondirected wireless infrared indoor diffuse link. Electronics and Communication in Japan, 9-19.

He, X., Cao, G., \& Zou, N. (2011). Simulation of White Light Based on Mixed RGB LEDs. IET International Conference on Communication Technology and Application. Beijing.

Kahn, J., \& Barry, J. (1997). Wireless Infrared Communications. Proceedings of IEEE.

Kahn, J., Krause, W., \& Carruthers, J. B. (1995). Experimental Characterization of Non directed Indoor Infrared Channels. IEEE Transactions on Communications, 40(234), 16131623.

Lee, K., Park, H., \& Barry, J. (2011). Indoor Channel Characteristics for Visible Light Communications. IEEE Communications Letters, 15(2), 217-219.

Lomba, C., Valadas, R., \& Duarte, A. (2000). Efficient simulation of the impulse response of the indoor wireless optical channel. International Journal of Communication Systems, 537-549.

Marcus, M., \& Pattan, B. (2005). Millimeter Wave Propagation: Spectrum Management Implications. IEEE Microwave Magazine, 6(2), 54-62.

Moreira, A., Valadas, R., \& Duarte, A. (1997). Optical interference produced by artificial light. Wireless Networks, $131-140$

Moreira, A., Valadas, R., \& Duarte, A. (1997). Optical Interference produced by artificial light. Wireless Networks, 3(2), 131-140.

Narasimhan, R., Audeh, M., \& Kahn, J. (1996). Effect of electronic-ballast fluorescent lighting on wireless infrared links. IEE Proceedings-Optoelectronics, 143, 347-354.

Ramaswami, R. (2002). Optical Fiber Communication: From Transmission to Networking. IEEE Communications Magazine, 40(5), 138-147.

Ricklin, J., Hammel, S., Eaton, F., \& Lachinova, S. (2006). Atmospheric channel effects on free space laser communication. Journal of Optical and Fiber Communications Research, 111-156.

Song, M., Pincemin, E., Vgenopoulou, V., Roudas, I., Amhoud, E., \& Jaouen, Y. (2015). Transmission performances of 400 Gbps coherent 16-QAM multi-band OFDM adopting nonlinear mitigation techniques. 2015 Tyrrhenian International Workshop on Digital Communications. Florence.

Street, M., Stavrinou, P., Obrien, D., \& Edwards, D. (1997). Indoor optical wireless systems. Optical and Quantum Electronics, 349-378.
Tekin, M., Savaşcıhabeş, A., \& Ertuğ, Ö. (2021). M-CSK-FlipOFDM for Visible Light Communication Systems. 44th International Conference on Telecommunications and Signal Processing, (s. 106-109). Brno, Czech Republic.

Xu, X. (2015). Advanced Modulation Formats for 400-Gbps Short-reach Optical Inter-Connection. Optics Express, 23(1), 492-500.

Zhang, S. (2017). Capacity-Approaching Transmission over $6375 \mathrm{~km}$ Using Hybrid Quasi-Single-Mode fiber Spans. Journal of Lightwave Technology, 35(3), 481-487. 